\title{
A dynamical classification of the range of pair interactions
}

\author{
A. Gabrielli ${ }^{1,2}$, M. Joyce ${ }^{3,4}$, B. Marcos ${ }^{5}$ and F. Sicard ${ }^{3}$ \\ ${ }^{1}$ SMC, CNR-INFM, Physics Department, University "Sapienza" of Rome, Piazzale Aldo Moro 2, 00185-Rome, Italy \\ ${ }^{2}$ Istituto dei Sistemi Complessi - CNR, Via dei Taurini 19, 00185-Rome, Italy \\ ${ }^{3}$ Laboratoire de Physique Nucléaire et Hautes Énergies, \\ Université Pierre et Marie Curie - Paris 6, CNRS IN2P3 UMR 7585, 4 Place Jussieu, 75752 Paris Cedex 05, France \\ ${ }^{4}$ Laboratoire de Physique Théorique de la Matière Condensée, \\ Université Pierre et Marie Curie - Paris 6, CNRS UMR 7600, \\ 4 Place Jussieu, 75752 Paris Cedex 05, France and \\ ${ }^{5}$ Laboratoire J.-A. Dieudonné, UMR 6621, Université de Nice - Sophia Antipolis, Parc Valrose 06108 Nice Cedex 02, France
}

\begin{abstract}
We formalize a classification of pair interactions based on the convergence properties of the forces acting on particles as a function of system size. We do so by considering the behavior of the probability distribution function (PDF) $P(\mathbf{F})$ of the force field $\mathbf{F}$ in a particle distribution in the limit that the size of the system is taken to infinity at constant particle density, i.e., in the "usual" thermodynamic limit. For a pair interaction potential $V(r)$ with $V(r \rightarrow \infty) \sim 1 / r^{\gamma}$ defining a bounded pair force, we show that $P(\mathbf{F})$ converges continuously to a well-defined and rapidly decreasing PDF if and only if the pair force is absolutely integrable, i.e., for $\gamma>d-1$, where $d$ is the spatial dimension. We refer to this case as dynamically short-range, because the dominant contribution to the force on a typical particle in this limit arises from particles in a finite neighborhood around it. For the dynamically long-range case, i.e., $\gamma \leq d-1$, on the other hand, the dominant contribution to the force comes from the mean field due to the bulk, which becomes undefined in this limit. We discuss also how, for $\gamma \leq d-1$ (and notably, for the case of gravity, $\gamma=d-2) P(\mathbf{F})$ may, in some cases, be defined in a weaker sense. This involves a regularization of the force summation which is generalization of the procedure employed to define gravitational forces in an infinite static homogeneous universe. We explain that the relevant classification in this context is, however, that which divides pair forces with $\gamma>d-2$ (or $\gamma<d-2$ ), for which the PDF of the difference in forces is defined (or not defined) in the infinite system limit, without any regularization. In the former case dynamics can, as for the (marginal) case of gravity, be defined consistently in an infinite uniform system.
\end{abstract}

PACS numbers: 98.80.-k, 05.70.-a, 02.50.-r, 05.40.-a

\section{INTRODUCTION}

Interactions are traditionally classified as long-range (or short-range) with respect to the non-additivity (or additivity) of the potential energy in the usual thermodynamic limit, i.e., when the number of particles $N$ and volume $V$ are taken to infinity at constant particle density. This is the property which determines the way in which standard instruments of statistical mechanics are applied to determine equilibrium properties (see e.g. [1-3]). Indeed in the case of long-range interactions, these instruments are applied using an appropriately generalized thermodynamic limit, in which the coupling or density are also scaled with system size. Such an analysis gives rise generically to features at equilibrium which are qualitatively different from those in shortrange systems - inhomogeneous statistical equilibria, non-equivalence of statistical ensembles, negative specific heat in the microcanonical ensemble (see e.g. [2, 3]). Most of these unusual features were first noted and studied in the context of the study of gravitating systems in astrophysics (see e.g. [4, 5] for reviews), and it has been realized in recent years that they are more generic in long-range interacting systems. This thermodynamic analysis extended to long-range systems is believed to determine, however, the behavior of such systems only on time scales which diverge as some power of $N$ (when expressed in terms of the characteristic dynamical time scales). On shorter times scales - usually those of interest in practical applications - study of several such systems (see e.g. [6 6 10] and references therein) shows that they appear generically, like in the well-documented case of gravity, to relax from almost any initial conditions to (almost) time-independent states - referred to variously as "meta-equilibria", "quasi-equilibria" or "quasistationary states" (QSS). The physics of these states, which are generically very different from those at thermal equilibrium, is understood to be the result of evolution in the collisionless regime described by Vlasov equation (usually referred to as the "collisionless Boltzmann equation" in the astrophysical literature [11]). Both the genesis of these states and their long-time relaxation are poorly understood, and are the subject of active study (see e.g. [12 16]).

In this article we consider a simple classification of pair interactions different to this usual thermodynamic one. Instead of considering the convergence properties of potential energy in the usual thermodynamic limit, we consider those of the force, in the same limit. The resulting classification can, like the usual one, be under- 
stood easily from simple considerations. To see this let us consider, as illustrated schematically in Fig. 1, a uni-

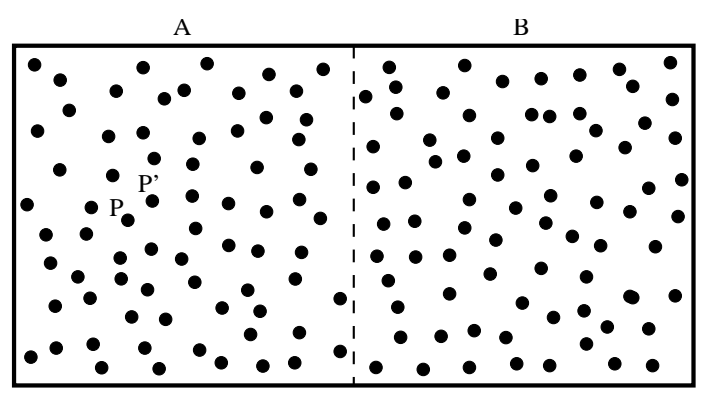

FIG. 1: An (approximately) uniform system consisting of two sub-systems A and B.

form system of particles interacting by a pair potential $V(r \rightarrow \infty) \sim 1 / r^{\gamma}$, and divided into two pieces, $A$ and $B$. For the usual thermodynamic classification one can consider that when the potential $V(r)$ is integrable at large $r$, i.e., $\gamma>d$ where $d$ is the spatial dimension, the potential energy of a typical particle comes essentially from its interaction with particles in a finite region about it. The energy of a particle (e.g. $P$ or $P^{\prime}$ ) in $A$ is thus insensitive to whether $B$ is present or not (and thus the total energy is equal, up to surface effects, to the sum of the energies of the subsystems): from an energetic point of view a particle "does not care" what the size of the system is, and the interaction is in this sense short-range. The distinction we consider is the analogous one deduced when one reasons in terms of force (or acceleration) rather than potential energy, and since forces are the primary physical quantities in dynamics, we refer to the corresponding classification as one of dynamical range. It is straightforward to see that in such a system, if the pair force is absolutely integrable, i.e., $\gamma>d-1$, the force acting on a typical particle is due essentially to its interaction with particles in a finite neighborhood around it, while if $\gamma<d-1$ this is not the case. Thus in the former case a particle in $A$ "does not care" whether the sub-system $B$ is present or not, and in this sense the interaction is "dynamically short-range". The classification differs from the standard one for interactions with $d<\gamma<d-1$ : for such interactions the potential energy "sees the bulk", but the force, which is its derivative, does not.

While the principle motivation for defining such a classification is that it may be relevant to understanding the qualitative behaviors of the out of equilibrium dynamics of such systems, it is not the aim of this article to establish that this is the case. We will limit ourselves in this respect to some brief remarks in our conclusions below. Our goal here is to provide a precise formulation of such a classification of the range of interactions based on the convergence properties of forces. While in the usual thermodynamic classification case one considers (see e.g.[1]) the mathematical properties of essential functions describing systems at equilibrium in the limit $N \rightarrow \infty, V \rightarrow \infty$ at fixed particle density $n_{0}=N / V$ (i.e. the usual thermodynamic limit), we will consider the behavior of functions characterising the forces in this same limit. More specifically we consider, following an approach introduced by Chandrasekhar for the case of gravity [17, 18], the definedness of the probability distribution function (PDF) of the force field in statistically homogeneous particle distributions as the size of the system becomes arbitrarily large. Such distributions and this limit may be described mathematically using the language of stochastic point processes, considering the class of such processes which have a well defined positive mean density when the infinite size limit is considered. To avoid any confusion we will refer to the usual thermodynamic limit in this context simply as the infinite system limit. Indeed the existence or non-existence of the quantities we are studying in this limit has no direct relation here to the determination of properties at thermal equilibrium. Further, in the context of the literature on long-range interactions the term "thermodynamic limit" is now widely associated with the generalized such limit, which involves adopting a different scaling of $V$ (or possibly coupling constants) with $N$ (for a discussion see e.g. [19]).

In this article we also discuss, in Sec. IV] a further (and different) classification which can be given of pair interactions according to their range. This is relevant when one addresses more generally, for any given pair interaction, a question which arises for Newtonian gravity in a cosmological setting: can a consistent dynamics be defined in an infinite system with non-zero density? A rigorous approach to the same question and the connection with the possibility of defining a statistical mechanical state for the system has been developed in [20, 21] in the particular case of a short range non-negative pair potential with finite support. Our conclusion, which generalizes a previous discussion given by two of us in [22], is that the answer to this question is that a necessary and sufficient condition for such a dynamics is not the integrability of the pair force, but instead the integrability of its gradient. This means that one requires $\gamma \geq d-2$, with gravity in any dimension (i.e. $\gamma=d-2$, the interaction potential solving the appropriate $d$-dimensional Poisson equation) being the marginal case in which such an infinite system limit may be defined. The reason is simply that, in an infinite system without any preferred point (i.e. when this limit is defined respecting statistical translational invariance), the physically meaningful quantity is the relative position of particles (as there is no meaning to absolute position). It is thus the convergence of relative forces on particles with system size which matters. In terms of the schema given above the distinction arises thus when one considers two close-by points (e.g. $P$ and $P^{\prime}$ in Fig. 1) in sub-system $A$, say, and asks whether their relative forces - and thus relative motions - depend on the presence of $B$ or not (or, equivalently, on the size of the system). The answer is that this difference of forces does not essentially depend on $B$ if the gradient of the pair force is absolutely integrable, i.e., $\gamma>d-2$, as in this case this 
difference is dominated by the contribution from particles in a finite neighborhood around them. Thus for the case that $d-2<\gamma \leq d-1$ the forces acting on two such particles become ill defined as the size of the system is extended to infinity, but their difference remains finite. Indeed, as has been discussed in [22] in the context of gravity in one dimension, the diverging component of the force on a particle represents a force on their centre of mass, which has no physical relevance in an infinite system without a preferred origin.

The paper is organized as follows. In the next section we recall the essential properties of stochastic point processes of relevance to our considerations, and then consider the general analyticity properties of the PDF of the total force at an arbitrary spatial point in such a particle distribution. We show that, for any pair force which is bounded, this PDF in the infinite volume limit is either well defined and rapidly decreasing, or else vanishes pointwise, i.e., the total force is an ill defined stochastic quantity. This means that it suffices, when studying pair potentials with different possible behaviors at large scales, to show that some chosen moment of the PDF converges to a finite value in this limit (or diverges) in order to establish that the whole PDF itself is well-defined (or ill defined). In Sec. III we give a general and formal expression for the variance of the total force PDF in a generic infinite uniform stochastic process in terms of the pair force and the two-point correlation properties of the SPP. From this we then deduce our principal result that the force $\mathrm{PDF}$ exists strictly in the infinite system limit if and only if the pair force is absolutely integrable at large separations (i.e. $\gamma \leq d-1$ ), while it can be defined only in a weaker sense, introducing a regularization, when the pair force is not absolutely integrable. In the following section we discuss the physical relevance of the use of such a regularization, which is the generalization of a simple formulation given by Kiessling [23] of that originally introduced by Jeans for the case of gravity [24], often misleadingly referred to as the "Jeans swindle" [11, 23]. By analyzing the evolution of density perturbations in an infinite system, we show that the physical relevance of such a regularization of the forces requires also a constraint on the behavior of the PDF of total force differences as a function of system size. This leads to the conclusion that $\gamma \leq d-2$ is the necessary and sufficient condition in order for it to be possible to have a well defined infinite system limit at constant density for dynamics under a given pair interaction. In the conclusions we review briefly the the relation of our results to previous work in the literature, and comment a little more on the possible relevance of our principle classification of interactions into dynamically short-range and dynamically long-range to the study of the out of equilibrium dynamics of such systems.

\section{THE FORCE PDF IN UNIFORM STOCHASTIC POINT PROCESSES: GENERAL RESULTS}

We first recall the definitions of some basic quantities used in the statistical characterization of a stochastic point process and define the total force PDF (see e.g. [18] for a detailed discussion). We then derive some results on the analyticity properties of the latter quantity which we will exploit in deriving our central results in the next section.

\section{A. Stochastic point processes}

In order to study the properties of the force field in the infinite system limit given by $N \rightarrow \infty, V \rightarrow \infty$ with fixed average density $n_{0}>0$ for a large scale uniform and spatially homogeneous particle system, we generalize the approach introduced by Chandrasekhar in [17] for the total gravitational field in a homogeneous Poisson particle distribution to more general cases and spatial dimensions. To do so we need to characterize statistically point-particle distributions in this limit, and we do this using the language of stochastic point processes (SPP). The microscopic number density of a single realization of the process is

$$
n(\mathbf{x})=\sum_{i} \delta\left(\mathbf{x}-\mathbf{x}_{i}\right)
$$

where $\delta$ is the $d$-dimensional Dirac delta function, $\mathbf{x}_{i}$ is the position of the $i^{\text {th }}$ system particle and the sum runs over all the particles of the system. We will limit our discussion to particle distributions in a euclidean $d$-dimensional space which are (i) statistically translationally invariant (i.e. spatially homogeneous or stationary) and (ii) large scale uniform in the infinite volume limit. Property (i) means that the statistical properties around a given spatial point of the particle distribution do not depend on the location of the point. In other words the statistical weights of two realizations of the point process, of which one is the rigidly translated version of the other, are the same and do not depend on the translation vector. In particular this implies that the ensemble average (i.e. average over the realizations of the $\mathrm{SPP})\langle n(\mathbf{x})\rangle$ of the microscopic number density takes a constant value $n_{0}>0$ independent of $\mathbf{x}$. Moreover the two-point correlation function of the microscopic density $\left\langle n(\mathbf{x}) n\left(\mathbf{x}^{\prime}\right)\right\rangle$ depends only on the vector distance $\mathbf{x}-\mathbf{x}^{\prime}$. Feature (ii) means that the average particle number fluctuation $\delta N(R)=\left(\left\langle N^{2}(R)\right\rangle-\langle N(R)\rangle^{2}\right)^{1 / 2}$ in a sphere of radius $R$ increases slower with $R$ than the average number $\langle N(R)\rangle_{0} V(R)$ with $R$, where $V(R) \propto R^{d}$ is the volume of the $d$-dimensional sphere.

Let us start by considering a generic realization of the particle distribution in a finite volume $V$ and let the total number of particles of the given realization be $N$. The particle positions $\mathbf{x}_{i}$ are fully characterized statistically 
by the joint probability densty function $(\mathrm{PDF}) \mathcal{P}_{N}\left(\left\{\mathbf{x}_{i}\right\}\right)$ conditional to having $N$ particles in the realization $\left(\left\{\mathbf{x}_{i}\right\}\right.$ indicates the set of positions of all system particles in the given realization). As a simple, but paradigmatic example we can think of the homogeneous $d$-dimensional Poisson point process. In this case $\mathcal{P}_{N}\left(\left\{\mathbf{x}_{i}\right\}\right)=V^{-N}$ simply and independently of the value of $n_{0}$. Given a function $X\left(\left\{\mathbf{x}_{i}\right\}\right)$ of the $N$ particle positions in thevolume $V$ its average, conditional to the value of $N$, can be written as

$$
\langle X\rangle_{N} \equiv \int_{V}\left[\prod_{i=1}^{N} d^{d} x_{i}\right] \mathcal{P}_{N}\left(\left\{\mathbf{x}_{i}\right\}\right) X\left(\left\{\mathbf{x}_{i}\right\}\right),
$$

where the position of each particle is integrated in the volume $V$. In order to evaluate the unconditional average of the property $X$, for which all possible outcomes of the value $N$ are considered, one would need the probability $q_{N}$ of having $N$ particles in the volume $V$, which permits to write:

$$
\langle X\rangle=\sum_{N=0}^{\infty} q_{N}\langle X\rangle_{N}
$$

in a strict analogy with the grand canonical ensemble average in equilibrium statistical mechanics. However, since we are restricting the discussion to large scale uniform particle distributions, for which $\delta N(R) /\langle N(R)\rangle$ vanishes for asymptotically large $R$, we expect that the larger the volume $V$ the narrower will be the peak around $N=\langle N(V)\rangle=n_{0} V$ in which the measure $q_{N}$ will be concentrated (for simplicity we have indicated with $V$ both the region and its size). Asymptotically we expect that only the term of index $N_{0} V$ will contribute to the sum in Eq. (2), i.e., for sufficiently large $V$ we can write:

$$
\langle X\rangle \simeq\langle X\rangle_{N_{0} V} \text {. }
$$

In other words we can consider that for sufficiently large $V$ the conditional PDF $\mathcal{P}_{n_{0} V}\left(\left\{\mathbf{x}_{i}\right\}\right)$ characterizes completely the statistical properties of the particle distribution in the finite volume $V$ and use this to evaluate in the following subsection the statistical properties of the total force. This is exactly what has been done, for instance, by Chandrasekhar in 17 to calculate the total gravitational force PDF in the Poissonian case.

In Appendix $\mathrm{A}$ we recall some of the basic definitions and properties of the statistical characterizations of uniform SPP. We will use below notably two essential properties of $S(\mathbf{k})$, the structure factor (SF), which follow from its definition:

$$
\lim _{k \rightarrow 0} k^{d} S(\mathbf{k})=0,
$$

i.e, the $\mathrm{SF}$ is an integrable function of $\mathbf{k}$ at $k=0$, and

$$
\lim _{k \rightarrow \infty} S(\mathbf{k})=1
$$

\section{B. General expression for the force PDF}

Let us consider now that the particles in any realization of the SPP interact through a pair force $\mathbf{f}(\mathbf{x})$, i.e., $\mathbf{f}(\mathbf{x})$ is the force exerted by a particle on another one at vectorial separation $\mathbf{x}$. Further we will assume that the pair force is

- central, i.e.,

$$
\mathbf{f}(\mathbf{x})=\hat{\mathbf{x}} f(x),
$$

where $\hat{\mathbf{x}}=\mathbf{x} / x$, and

- bounded, i.e., there exists $f_{0}<\infty$ such that $|\mathbf{f}(\mathbf{x})|=$ $f(x) \leq f_{0}$ for all $\mathbf{x}$.

These assumptions simplify our calculations considerably, but do not limit our aim which is to establish the relation solely between the statistical properties of the force field and the behavior of the pair interaction at large distances. Note that the second assumption means that, in cases such as the gravitational or the Coulomb interaction, the divergence at zero separation is assumed appropriately regularized. We will briefly describe in our conclusions below how our results could be generalized to include such singularities.

Let us assume for the moment that the system volume $V$ is finite. As shown above, if $V$ is sufficiently large, one can consider that the number of particles in this volume is deterministically $N_{0} V$. We will deal with the important problem of the infinite volume limit defined by $N, V \rightarrow \infty$ with $N / V \rightarrow n_{0}>0$ in the next subsection, by studying directly the limit $V \rightarrow \infty$ with fixed $N_{0} V$. The total force field $\mathbf{F}(\mathbf{x})$ at a point $\mathbf{x}$, i.e., the force on a test particle placed at a point $\mathbf{x}$, may thus be written

$$
\mathbf{F}(\mathbf{x})=\sum_{i=1}^{N} \mathbf{f}\left(\mathbf{x}-\mathbf{x}_{i}\right)=\sum_{i=1}^{N} \frac{\mathbf{x}-\mathbf{x}_{i}}{\left|\mathbf{x}-\mathbf{x}_{i}\right|} f\left(\left|\mathbf{x}-\mathbf{x}_{i}\right|\right) .
$$

The force field $\mathbf{F}(\mathbf{x})$ may be considered as a stochastic variable with respect to the SPP. Choosing arbitrarily the origin as the point where the total force is evaluated, the PDF of this force is formally defined by ${ }^{1}$

$$
P_{N}(\mathbf{F})=\int_{V}\left[\prod_{i=1}^{N} d^{d} x_{i}\right] \mathcal{P}_{N}\left(\left\{\mathbf{x}_{i}\right\}\right) \delta\left[\mathbf{F}+\sum_{i} \mathbf{f}\left(\mathbf{x}_{i}\right)\right],
$$

\footnotetext{
${ }^{1}$ We consider here the unconditional force PDF, i.e., the force is that at an arbitrary spatial point, rather than that on a point occupied by a particle which belongs to the particle distribution. It is the latter case, of the conditional force PDF, which is often considered in calculations of this kind (see e.g. [25-27]). The distinction is not important here as the constraints we derive, which depend on the large scale correlation properties of the particle distribution, would be expected to be the same in both cases.
} 
where we have used, as assumed, that $\mathbf{f}\left(-\mathbf{x}_{i}\right)=-\mathbf{f}\left(\mathbf{x}_{i}\right)$. Using the identity

$$
\delta(\mathbf{y})=\frac{1}{(2 \pi)^{d}} \int d^{d} q e^{i \mathbf{q} \cdot \mathbf{y}}
$$

this can be rewritten as

$P_{N}(\mathbf{F})=\frac{1}{(2 \pi)^{d}} \int d^{d} q e^{i \mathbf{q} \cdot \mathbf{F}} \int_{V}\left[\prod_{i=1}^{N} d^{d} x_{i} e^{i \mathbf{q} \cdot \mathbf{f}\left(\mathbf{x}_{i}\right)}\right] \mathcal{P}_{N}\left(\left\{\mathbf{x}_{i}\right\}\right)$

The integral over the spatial coordinates in the above equation defines the characteristic function of the total field $\mathbf{F}$

$$
\tilde{P}_{N}(\mathbf{q})=\int_{V}\left[\prod_{i=1}^{N} d^{d} x_{i} e^{i \mathbf{q} \cdot \mathbf{f}\left(\mathbf{x}_{i}\right)}\right] \mathcal{P}_{N}\left(\left\{\mathbf{x}_{i}\right\}\right),
$$

so that

$$
P_{N}(\mathbf{F})=\frac{1}{(2 \pi)^{d}} \int d^{d} q e^{i \mathbf{q} \cdot \mathbf{F}} \tilde{P}_{N}(\mathbf{q}) .
$$

The integral over spatial configurations in Eq. (8) can be conveniently rewritten as an integral over the possible values of the pair forces due to each of the $i=1, \ldots, N$ particles:

$$
\tilde{P}_{N}(\mathbf{q}) \equiv \int\left[\prod_{i=1}^{N} d^{d} f_{i} e^{i \mathbf{q} \cdot \mathbf{f}_{i}}\right] \mathcal{Q}_{N}\left(\left\{\mathbf{f}_{i}\right\}\right)
$$

where

$$
\mathcal{Q}_{N}\left(\left\{\mathbf{f}_{i}\right\}\right)=\int_{V}\left[\prod_{i=1}^{N} d^{d} x_{i}\right] \mathcal{P}_{N}\left(\left\{\mathbf{x}_{i}\right\}\right) \prod_{i=1}^{N} \delta\left[\mathbf{f}_{i}-\mathbf{f}\left(\mathbf{x}_{i}\right)\right]
$$

is the joint PDF for the pair forces $\mathbf{f}_{i}$. Note that, since $\mathbf{F}$ is the sum of the variables $\left\{\mathbf{f}_{i}\right\}$ its characteristic function $\tilde{P}_{N}(\mathbf{q})$ can be given as

$$
\tilde{P}_{N}(\mathbf{q})=\tilde{\mathcal{Q}}_{N}\left(\left\{\mathbf{q}_{i}=\mathbf{q}\right\}\right)
$$

where $\tilde{\mathcal{Q}}_{N}\left(\left\{\mathbf{q}_{i}\right\}\right)$ is the $N d$-dimensional FT of the joint pair forces PDF $\mathcal{Q}_{N}\left(\left\{\mathbf{f}_{i}\right\}\right)$, i.e.,

$$
\tilde{\mathcal{Q}}_{N}\left(\left\{\mathbf{q}_{i}\right\}\right)=\int\left[\prod_{i=1}^{N} d^{d} f_{i} e^{i \mathbf{q}_{i} \cdot \mathbf{f}_{i}}\right] \mathcal{Q}_{N}\left(\left\{\mathbf{f}_{i}\right\}\right)
$$

\section{Analyticity properties of the force PDF}

From the fact that the pair force is bounded it follows that $\mathcal{Q}_{N}\left(\left\{\mathbf{f}_{i}\right\}\right)$ has a compact support, and, since it is absolutely integrable (by definition), FT theory (see e.g. [28]) implies that its characteristic function $\tilde{\mathcal{Q}}_{N}\left(\left\{\mathbf{q}_{i}\right\}\right)$ is an analytic function of the variables $\left\{\mathbf{q}_{i}\right\}$. Consequently
$\tilde{P}_{N}(\mathbf{q})$ is an analytic function of $\mathbf{q}$. Again from FT theory one has therefore that $P_{N}(\mathbf{F})$ is a rapidly decreasing function of $\mathbf{F}$ :

$$
\lim _{F \rightarrow \infty} F^{\alpha} P_{N}(\mathbf{F})=0, \quad \forall \alpha>0 .
$$

Thus $P_{N}(\mathbf{F})$ is a well-defined function of which all moments finite, i.e., $0<\left\langle|\mathbf{F}|^{n}\right\rangle<+\infty$ for any $n \geq 0$.

Let us now consider what happens when we take the limit $V \rightarrow \infty$ with $N_{0} V$. On one hand the joint PDF $\mathcal{Q}_{N}\left(\left\{\mathbf{f}_{i}\right\}\right)$ remains non-negative and absolutely integrable at all increasing $V$. On the other hand the support of this function remains compact with a diameter unaffected by the values of $V$, but fixed only by $f_{0}$. Therefore we expect that the FT theorem keeps its validity also in the infinite system limit resulting in an analytical

$$
\tilde{P}(\mathbf{q}) \equiv \lim _{\substack{V \rightarrow \infty \\ N / V_{0}}} \tilde{P}_{N}(\mathbf{q})
$$

Therefore we will have that

$$
P(\mathbf{F}) \equiv \lim _{\substack{V \rightarrow \infty \\ N_{0} V}} P_{N}(\mathbf{F})
$$

satisfies

$$
\lim _{F \rightarrow \infty} F^{\alpha} P(\mathbf{F})=0, \quad \forall \alpha>0 .
$$

There are then only two possibilities for the behavior of $\tilde{P}_{N}(\mathbf{q})$ in the infinite system limit:

1. It converges to an absolutely integrable function which is not identically zero everywhere, giving a $P(\mathbf{F})$ which is normalizable and non-negative on its support. Further all the integer moments of $|\mathbf{F}|$ are positive and finite.

2. It converges to zero everywhere, giving $P(\mathbf{F}) \equiv$ 0 . More specifically $P_{N}(\mathbf{F})$ with $N_{0} V$ converges point-wise to the null function: it becomes broader and broader with increasing $N$ (and $V$ ), but with an amplitude which decreases correspondingly and eventually goes to zero in the limit.

This latter case is analogous to the case of the sum of identically distributed uncorrelated random variables: if this sum is not normalized with the appropriate power of the number $N$ of such variables, the PDF of the sum vanishes point-wise in a similar way in the limit $N \rightarrow \infty$.

In summary it follows from these considerations of the analyticity properties of $\tilde{P}_{N}(\mathbf{q})$ at increasing $V$ that the case of a well defined, but fat tailed $P(\mathbf{F})$, can be excluded: in the infinite system limit the force PDF, if defined, is expected to be a normalizable and rapidly decreasing function. 


\section{LARGE DISTANCE BEHAVIOR OF PAIR INTERACTIONS AND THE FORCE PDF}

In this section we use the result derived in the previous section to infer the main result of this paper: the relation between the large scale behavior of the pair interaction and the force PDF in the infinite system limit.

We thus consider, as above, a central and bounded pair force such that

$$
f(x) \simeq \frac{g}{x^{\gamma+1}} \text { for } x \rightarrow \infty,
$$

or, equivalently, a pair interaction corresponding to a two-body potential $V(x) \simeq g /\left(\gamma x^{\gamma}\right)$ at large $x$ for $\gamma \neq 0$ (and from $V(x) \simeq-g \ln x$ for $\gamma=0$ ). Since the pair force is bounded, we have $\gamma>-1$.

Given the final result derived in the previous section, it follows that, to determine whether the force PDF exists, it is sufficient to analyze a single even moment of this PDF: because the PDF, when it exists, is rapidly decreasing, any such moment is necessarily finite and non-zero in this case, and diverges instead when the PDF does not exist. We choose to analyze the behavior of the second moment, $\left\langle F^{2}\right\rangle$, which is equal to the variance of the PDF since the first moment $\langle\mathbf{F}\rangle$ is zero (see below). We choose this moment because, as we will now see, it can be expressed solely in terms of the FT of $\mathbf{f}(\mathbf{x})$ and of the SF of the microscopic density of the particle distribution. From these expressions we can then infer easily our result.

\section{A. Variance of the force in infinite system limit}

The formal expression of the total force acting on a test particle (i.e. the force field) at $\mathbf{x}$ in the infinite system limit may be written

$$
\mathbf{F}(\mathbf{x})=\int d^{d} x^{\prime} \frac{\mathbf{x}-\mathbf{x}^{\prime}}{\left|\mathbf{x}-\mathbf{x}^{\prime}\right|} f\left(\left|\mathbf{x}-\mathbf{x}^{\prime}\right|\right) n\left(\mathbf{x}^{\prime}\right)
$$

where the integral is over the infinite space and $n(\mathbf{x})$, given in Eq. (11), is the density field in a realization of the general class of uniform SPP we have discussed with positive mean density $n_{0}$.

It is simple to show, using Eq. (14) and the definition of the SF given above in Eq. (A1), that formally

$$
\left\langle\mathbf{F}^{2}\right\rangle=\frac{1}{(2 \pi)^{d}} \int d^{d} k|\tilde{\mathbf{f}}(\mathbf{k})|^{2} S(k)
$$

where $\tilde{\mathbf{f}}(\mathbf{k})$ is the (d-dimensional) FT of $\hat{\mathbf{x}} f(x)$. It is straightforward to show that $\tilde{\mathbf{f}}(\mathbf{k})=\hat{\mathbf{k}} \tilde{f}(k)$, where the explicit expression for $\tilde{f}(k)$ is given in the appendix ${ }^{2}$.

\footnotetext{
2 Note that only in $d=1$ does $\tilde{f}(k)$ coincide with the direct FT of $f(x)$.
}

We can thus write

$$
\begin{aligned}
\left\langle\mathbf{F}^{2}\right\rangle & =\frac{1}{(2 \pi)^{d}} \int d^{d} k|\tilde{f}(k)|^{2} S(k) \\
& =\frac{1}{2^{d-1} \pi^{d / 2} \Gamma(d / 2)} \int_{0}^{\infty} d k k^{d-1}|\tilde{f}(k)|^{2} S(k),
\end{aligned}
$$

where $\Gamma(x)$ is the usual Euler Gamma function.

\section{B. Force PDF for an integrable pair force}

Let us now consider the integrability of the integrand in Eq. (16). We start with the case in which $f(x)$ is not only bounded but integrable in $\mathbb{R}^{d}$, i.e., with $\gamma>d-1$. Given these properties, it is straightforward to verify, using the conditions (3) and (4) on $S(k)$ and standard FT theorems, that the function $\left.|\tilde{f}(k)|^{2} S(k)\right)$ is also integrable in $\mathbb{R}^{d}$. The variance is therefore finite, from which it follows that the PDF exists, and furthermore that all its moments are finite.

\section{Force PDF for a non-integrable pair forces}

For a pair force which is absolutely non-integrable, i.e., $\gamma<d-1$, the FT $\tilde{\mathbf{f}}(\mathbf{k})$ of $\mathbf{f}(\mathbf{x})$ in Eq. (16) is defined only in the sense of distributions, i.e., the integrals over all space of $f(x)$ must be defined by a symmetric limiting procedure. Physically this means that the expression Eq. (14) for the force on a particle in infinite space must be calculated as

$\mathbf{F}(\mathbf{x})=\lim _{\mu \rightarrow 0^{+}} \lim _{V \rightarrow \infty} \int_{V} \frac{\mathbf{x}-\mathbf{x}^{\prime}}{\left|\mathbf{x}-\mathbf{x}^{\prime}\right|} f\left(\left|\mathbf{x}-\mathbf{x}^{\prime}\right|\right) e^{-\mu\left|\mathbf{x}-\mathbf{x}^{\prime}\right|} n\left(\mathbf{x}^{\prime}\right) d^{d} x^{\prime}$,

where the two limits do not commute. In other words, $\mathbf{F}(\mathbf{x})$ is defined as the zero screening limit of a screened version of the simple power law interaction in an infinite system. The expression Eq. (16) is then meaningful when $\tilde{f}(k)$ is taken to be defined in the analogous manner with the two limits $\mu \rightarrow 0^{+}$of the screening and $V \rightarrow \infty$ (i.e. with the minimal non-zero mode $k \sim 1 / V \rightarrow 0^{+}$) taken in the same order as indicated in Eq. (17).

Let us consider then again, for the case $\gamma<d-1$, the integrability of the integrand in Eq. (16). To do so we need to examine in detail the small $k$ behavior of $\tilde{f}(k)$. It is shown in the appendix that, as one would expect from a simple dimensional analysis, for $f(r \rightarrow \infty) \sim 1 / r^{\gamma+1}$ we have $f(k \rightarrow 0) \sim k^{-d+\gamma+1}$ in any $d$, for the case of a pair force which is not absolutely integrable, and bounded, i.e., $-1<\gamma<d-1$. It follows then from Eq. (16) that the variance is finite for a given $\gamma$ only for a sub-class of uniform point processes, specifically those which satisfy

$$
\lim _{k \rightarrow 0} k^{-d+2 \gamma+2} S(k)=0,
$$

i.e., for $S(k \rightarrow 0) \sim k^{n}$ with

$$
n>d-2 \gamma-2=-d+2(d-1-\gamma) .
$$


For uniform point processes violating this condition, i.e., with $S(k \rightarrow 0) \sim k^{n}$ and $-d<n \leq-d+2(d-\gamma-1)$, the variance diverges. It follows from the results on the PDF of $\mathbf{F}$ presented in the previous section that the total force itself $\mathbf{F}(\mathbf{x})$ is then badly defined in the infinite system limit.

These results of Sec. IIIB and Sec. IIIC combined are the central ones in this paper, anticipated in the introduction.

Firstly, when pair forces are absolutely integrable at large separations, the total force PDF is well defined in the infinite system limit, while for pair forces which are not absolutely integrable this quantity is ill defined. This has the simple physical meaning anticipated in the introduction: when this PDF is well defined, the force on a typical particle takes its dominant contribution from particles in a finite region around it; when instead the $\mathrm{PDF}$ is ill defined far-away contributions to the total force dominate, diverging with the size of the system. Thus absolutely integrable pair forces with $\gamma>d-1$ are, in this precise sense, "short-range", while they are "long-range" when $\gamma \leq d-1$. To avoid confusion with the usual classification of the range of interactions based on the integrability properties of the interaction potential, we will adopt the nomenclature that interactions in the case $\gamma>d-1$ are dynamically short-range, while for $\gamma \leq d-1$ they are dynamically long-range. Thus an interaction with $d-1<\gamma \leq d$ can be described as thermodynamically long-range but dynamically short-range.

Secondly the results in Sec. IIIC detail how, for $\gamma \leq$ $d-1$, the force PDF in the infinite system limit may be defined provided an additional prescription is given for the calculation of the force. In the next section we explain the physical meaning and relevance of this result.

\section{DEFINEDNESS OF DYNAMICS IN AN INFINITE UNIFORM SYSTEM}

The regularization Eq. (17) is simply the generalization to a generic pair force with $\gamma \leq d-1$ of one which is used for the case of Newtonian gravity, often referred to as the "Jeans swindle" (see e.g. 11]). It was indeed originally introduced by Jeans [24] in his treatment of self-gravitating matter in an infinite universe. However, as explained by Kiessling in [23], its denomination as a "swindle" is very misleading, as it can be formulated in a mathematically rigorous and physically meaningful manner, precisely as in Eq. (17).

The prescription Eq. (17) simply makes the force on a particle defined by setting to zero the ill defined contribution due to the non-zero mean density:

$$
\langle\mathbf{F}(\mathbf{x})\rangle=\lim _{\mu \rightarrow 0^{+}} n_{0} \int \frac{\mathbf{x}-\mathbf{x}^{\prime}}{\left|\mathbf{x}-\mathbf{x}^{\prime}\right|} f\left(\left|\mathbf{x}-\mathbf{x}^{\prime}\right|\right) e^{-\mu\left|\mathbf{x}-\mathbf{x}^{\prime}\right|} d^{d} x^{\prime}=0,
$$

The force on a particle can thus be written as

$$
\mathbf{F}(\mathbf{x})=\lim _{\mu \rightarrow 0^{+}} \int \frac{\mathbf{x}-\mathbf{x}^{\prime}}{\left|\mathbf{x}-\mathbf{x}^{\prime}\right|} f\left(\left|\mathbf{x}-\mathbf{x}^{\prime}\right|\right) e^{-\mu\left|\mathbf{x}-\mathbf{x}^{\prime}\right|} \delta n\left(\mathbf{x}^{\prime}\right) d^{d} x^{\prime},
$$

where $\delta n\left(\mathbf{x}^{\prime}\right)=n\left(\mathbf{x}^{\prime}\right)-n_{0}$ is the density fluctuation field. It is straightforward to show that the derived constraint (19) corresponds simply to that which can be anticipated by a naive analysis of the convergence of the integral Eq. (21): treating $\delta n\left(\mathbf{x}^{\prime}\right)$ as a deterministic function (rather than a stochastic field) one can require it to decay at large $\left|\mathbf{x}^{\prime}\right|$ with a sufficiently large exponent in order to give integrability; taking the FT to infer the behavior of $|\tilde{\delta} n(\mathbf{k})|^{2}$ one obtains the condition (19).

The relevance of the results we have derived for the force PDF in the infinite system limit using this regularization arises thus, as it does in the case of Newtonian gravity, when one addresses the following question: is it possible to define consistently dynamics under a given pair interaction in an infinite system which is uniform at large scales? As we now discuss, generalizing considerations given by two of us in [22] for the specific case of gravity in $d=1$, the answer to this question is in fact phrased in terms of the definedness of the PDF of force differences rather than that of forces. This leads then to our second classification of pair interactions.

\section{A. Evolution of fluctuations and definedness of PDF}

Let us consider first an infinite particle distribution which is such that the total force PDF is defined at some given time, i.e., for $\gamma>d-1$ we may consider any uniform SSP, while for $\gamma<d-1$ we may consider (employing the regularization discussed) only the class of SSP with fluctuations at large scales obeying the condition (19) at this time. The forces on particles at this initial time are then well defined. This will only remain true, however, after a finite time interval, if the evolved distribution continues to obey the same condition (19). Let us determine when this is the case or not.

In order to do so, it suffices to consider the evolution of the density fluctuations, and specifically of the SF at small $k$, due to the action of this force field. Given that we are interested in the long-wavelength modes of the density field, we can apply the differential form of the continuity equation for the mass (and thus number) density between an initial time $t=0$ and a time $t=\delta t$ :

$$
n(\mathbf{x}, \delta t)-n(\mathbf{x}, 0)=\vec{\nabla}[n(\mathbf{x}, 0) \mathbf{u}(\mathbf{x}, 0)]
$$

where $\mathbf{u}(\mathbf{x}, 0)$ is the infinitesimal displacement field. Subtracting the mean density $n_{0}$ from both sides, and linearizing in $\delta n(\mathbf{x}, \delta t)=\left[n(\mathbf{x}, \delta t)-n_{0}\right]$ and $\mathbf{u}(\mathbf{x}, 0)$, we obtain, on taking the FT,

$$
\tilde{\delta} n(\mathbf{k}, \delta t)=\tilde{\delta} n(\mathbf{k}, 0)+i n_{0} \mathbf{k} \cdot \tilde{\mathbf{u}}(\mathbf{k}, 0) .
$$


Taking the square modulus of both sides, in the same approximation we get

$$
\begin{aligned}
|\tilde{\delta} n(\mathbf{k}, \delta t)|^{2}- & |\tilde{\delta} n(\mathbf{k}, 0)|^{2}= \\
& n_{0}^{2} k^{2}|\tilde{\mathbf{u}}(\mathbf{k})|^{2}+2 \mathbf{k} n_{0} \operatorname{Im}\left[\tilde{\delta} n(\mathbf{k}, 0) \tilde{\mathbf{u}}^{*}(\mathbf{k}, 0)\right] .
\end{aligned}
$$

If the displacements are generated solely by the forces acting (i.e. assuming velocities are initially zero), we have that

$$
\mathbf{u}(\mathbf{x}, 0)=\frac{1}{2} \mathbf{F}(\mathbf{x}, 0) \delta t^{2}
$$

and thus, that $|\tilde{\mathbf{u}}(\mathbf{k})|^{2} \propto|\mathbf{F}(\mathbf{k})|^{2}$. The latter quantity is given, using Eq. (15), by

$$
|\mathbf{F}(\mathbf{k})|^{2}=|\tilde{f}(k)|^{2} S(k) .
$$

In the analysis in the previous section we used the result that at small $k, \tilde{f}(k) \sim k^{-d+\gamma+1}$. Thus $|\tilde{\mathbf{u}}(\mathbf{k})|^{2} \sim k^{2 m+n}$, where $m=-d+\gamma+1$, if $S(k) \sim k^{n}$. It then follows, from Eq. (24), that the small $k$ behavior of the timeevolved $\mathrm{SF}$ is given by

$$
S_{\delta t}(k \rightarrow 0) \sim k^{n}+k^{1+m+n}+k^{2+2 m+n} .
$$

It can be inferred that the leading small $k$ behavior of the $\mathrm{SF}$ is unchanged if and only if $m+1 \geq 0$, i.e., $\gamma \geq d-2$. Gravity $(\gamma=d-2)$ is the marginal case is which the long wavelength contribution to the SF generated by the evolution has the same exponent as the initial SF: this is the well known phenomenon of linear amplification of initial density perturbations (see e.g. [11, 29]) which applies ${ }^{3}$ in infinite self-gravitating systems (derived originally by Jeans).

If, on the other hand, $\gamma<d-2$ (i.e. the interaction is "more long-range" than gravity in $d$ dimensions) the exponent of the small $k$ behavior is reduced from $n$ to $n-2(d-2-\gamma)$. Given that our result is for an infinitesimal time $\delta t$, this indicates in fact a pathological behavior: in any finite time interval the exponent $n$ should become, apparently, arbitrarily large and negative, while, as shown in Sect. III, the constraint $n>-d$ is imposed by the assumed large scale uniformity of the SPP. In other words this result means that, in the infinite system limit, when $\gamma<d-2$, the condition of large scale uniformity is violated immediately by the dynamical evolution. The reason is simply that in this case the rate of growth of a perturbation at a given scale increases with the scale. Indeed this is the essential content of the analysis given just above: through the continuity equation, the perturbation to the density field is proportional

\footnotetext{
3 The result does not apply, however, when $n>4$ [29]; the reason is that fluctuations with $S(k \rightarrow 0) \sim k^{4}$ arise generically from any rearrangement of matter due to dynamics which conserves mass and momentum locally. These effects are neglected implicitly above when we use the continuum approximation to the density fluctuation field.
}

to the gradient of the displacement field, which in turn is simply proportional to the gradient of the force. As we now detail more explicitly, when $\gamma<d-2$, this quantity diverges with the size of the system.

\section{B. PDF of force differences}

Let us consider now the behavior of the PDF of the difference of the forces between two spatial points separated by a fixed vector distance a:

$$
\Delta \mathbf{F}(\mathbf{x} ; \mathbf{x}+\mathbf{a}) \equiv \mathbf{F}(\mathbf{x})-\mathbf{F}(\mathbf{x}+\mathbf{a})
$$

If this quantity is well defined in the infinite system limit, its PDF $\mathcal{P}(\boldsymbol{\Delta} \mathbf{F} ; a)$ will be independent of $\mathbf{x}$ and will have a parametric dependece only on $a=|\mathbf{a}|$ because of the assumed statistical translational and rotational invariance of the particle distribution.

The analysis of the properties of $\mathcal{P}(\boldsymbol{\Delta} \mathbf{F} ; a)$ in the infinite volume limit is formally exactly the same as that given above for the total force $\mathbf{F}$, with the only replacement of the pair force in Eq. (13) by the pair force difference:

$$
\Delta \mathbf{f}(\mathbf{x}, \mathbf{x}+\mathbf{a})=\mathbf{f}(\mathbf{x})-\mathbf{f}(\mathbf{x}+\mathbf{a})
$$

i.e., the difference of the pair forces on two points located at $\mathbf{x}$ and $\mathbf{x}+\mathbf{a}$ due to a point at the origin. Assuming again the possible small scale singularities in this pair force difference to be suitably regulated, our previous analysis carries through, the only significant change being that, as $x \rightarrow \infty$,

$$
\Delta \mathbf{f}(\mathbf{x}, \mathbf{x}+\mathbf{a}) \sim a \hat{\mathbf{x}} / x^{\gamma+2}
$$

Proceeding in exactly the same manner to analyse $\mathcal{P}(\Delta \mathbf{F} ; a)$, we find that

- For $\gamma>d-2$, i.e., if the gradient of the pair force at fixed $a$ is an absolutely integrable function of $\mathbf{x}$ at large separations, the $\operatorname{PDF} \mathcal{P}(\boldsymbol{\Delta} \mathbf{F} ; a)$ is well defined in the infinite system limit, and is a rapidly decreasing function of its argument for any SPP. This is true without any regularization.

- For $\gamma \leq d-2$, on the other hand, a well defined PDF may be obtained only by using the regularization like that introduced above in Eq. (17). Therefore the PDF of the force differences then remains well defined, i.e., the force difference $\Delta \mathbf{F}(\mathbf{x} ; \mathbf{a})$ remains finite at all $\mathbf{x}$, only in a sub-class of SPP defined by the constraint

$$
n>d-2 \gamma-4=-d+2(d-2-\gamma)
$$

For the case of gravity $\gamma=d-2$ this coincides with the full class of uniform SPP, while for any smaller $\gamma$, it restricts to a sub-class of the latter. 


\section{Conditions for definedness of dynamics in an infinite system}

Our analysis in Sec. IVA of the evolution of density perturbations under the effect of the mutual pair forces gave the sufficient condition $\gamma \geq d-2$ for the consistency of the dynamics in the infinite system limit, but with the assumption that the total force PDF was itself defined. This means that, in the range $d-2 \leq \gamma<d-1$, the result derived applies only to the sub-class of infinite uniform particle distributions in which the large scale fluctuations obey the condition (19). It is straightforward to verify, however, that the analysis and conclusions of Sec. IVA can be generalized to cover all uniform SPP for $\gamma \geq d-2$. In line with the discussion given above, the analysis requires in fact only assumptions about the behavior of the gradient of the forces, rather the forces themselves. More specifically, the only equation which explicitly contains the force, Eq. (25), is a purely formal step which can be modified to include the possibility that the force diverges with system size. Indeed if the force - at a given point - includes such a divergence it is sufficient that this divergence cancels out when we calculate the difference between this force and that at a neighboring point. Physically this means simply that, as discussed above, when we consider the relative motions of particles, it is sufficient to consider relative forces. Further, as we are considering the limit of an infinite system in which there is no preferred point (i.e. statistical homogeneity holds), only relative motions of points has physical significance, and therefore only the spatial variation of the forces can have physical meaning. These latter statements can be viewed as a kind of corollary to Mach's principle: if the mass distribution of the universe is, as it is in the case we consider, such that there is no preferred point in space (and, specifically, no center of mass) inertial frames which give absolute meaning to forces (rather than tidal forces) cannot be defined.

In summary our conclusion is that the necessary and sufficient condition for dynamics to be defined in the infinite system limit - in analogy to how it is defined for Newtonian self-gravitating particles in a infinite universe of constant density - is that the gradient of the pair force be absolutely integrable at large separations. Gravity is the marginal (logarithmically divergent) case in which such a dynamics can be defined, but only by using a prescription such as Eq. (17). Further these conditions on the range of pair forces can be expressed simply as one on the existence of the PDF of force differences of points as finite separations in the infinite system limit.

\section{DISCUSSION AND CONCLUSIONS}

In conclusion we make some brief remarks on how the results derived here relate to previous work in the literature on force PDFs. In this context we also discuss the important assumption we made throughout the arti- cle, that the pair force considered was bounded. Finally we return briefly to the question of the relevance of the classification dividing interactions according to the integrability properties of the pair force, concerning which we have reported initial results elsewhere [30].

The first and most known calculation of the force PDF is that of Chandrasekhar [17], who evaluated it for the gravitational pair interaction in an infinite homogeneous Poisson particle distribution (in $d=3$ ). This results in the so-called Holtzmark distribution, a probability distribution belonging to the Levy class (i.e. power law tailed with a diverging second moment) with $P(\mathbf{F}) \sim F^{-9 / 2}$ at large $F$. According to our results here, a well defined PDF may be obtained for such a force law, which is not absolutely integrable at large separations, only by using a prescription for the calculation of the force in the infinite system limit. In his calculation Chandrasekhar indeed obtains the force on a point by summing the contributions from mass in spheres of radius $R$ centered on the point considered, and then taking $R \rightarrow \infty$ (with $n_{0}$ fixed). This prescription is a slight variant of the one we have employed (following Kiessling 23]): instead of the smooth exponential screening of the interaction, it uses a "spherical top-hat" screening so that the force may be written formally as in Eq. (17) with the replacement of $e^{-\mu\left|\mathbf{x}-\mathbf{x}^{\prime}\right|}$ by a Heaviside function $\Theta\left(\mu^{-1}-\left|\mathbf{x}-\mathbf{x}^{\prime}\right|\right)$. It is straightforward to verify that the result of Chandrasekhar is unchanged if the smooth prescription Eq. (17) is used instead. As the Poisson distribution corresponds to an SF $S(k \rightarrow 0) \sim k^{n}$ with $n=0$, the general condition (19) for the existence of the PDF we have derived, which gives $n>-1$ for gravity in $d=3$, is indeed satisfied. The fact that the PDF is power-law tailed (and thus not rapidly decreasing) arises from the fact that the calculation of Chandrasekhar does not, as done here, assume that the singularity in the gravitational interaction is regularized. Indeed it is simple to show explicitly [18 that this power law tail arises from the divergence in the pair force at zero separation. This can be done by considering the contribution to the total force on a system particle due to its nearest neighbor particle, which turns out to have a power law tail identical, both in exponent and amplitude, to that of the full $P(\mathbf{F})$.

Our analysis shows that it is true in general that well defined, but power-law tailed force PDFs, can arise only when there are singularities in the pair force: for a bounded force we have seen that the PDF is necessarily rapidly decreasing when it exists. More specifically, returning to the analysis of Sec. IIC, it is straightforward to see that the crucial property we used of $\mathcal{Q}_{N}\left(\left\{\mathbf{f}_{i}\right\}\right)$, that it have compact support, is no longer valid when the pair force has singularities. The analyticity properties which lead to a rapidly decreasing PDF may then not be inferred. We note that this is true at finite $N$, and has nothing to do with the infinite volume limit, i.e., the appearance of the associated power-law tail arises from the possibility of having a single particle which give an 
unbounded contribution rather than from the combination of the contribution of many particles which then diverges in the infinite system limit. The exponent in such a power-law tail will depend on the nature of the divergence at small separation. More specifically, for a central pair force as considered above and now with a singularity $f(x \rightarrow 0) \sim 1 / x^{a}$, a simple generalization of the analysis for the case of gravity (see [18]) of the leading contribution to the total force coming from the nearest neighbor particle leads to the conclusion that $P(F \rightarrow \infty) \sim F^{-d-\frac{d}{a}}$ (where $\left.F=|\mathbf{F}|\right)$. This implies that the variance diverges (i.e. the PDF becomes fattailed) for $a>d / 2$.

Force PDFs have been calculated in various other specific cases. Wesenberg and Molmer [25] derived that of forces exerted by randomly distributed dipoles in $d=3$, corresponding to a pair force with $\gamma=2$. According to our results this is the marginal case in which a summation prescription is required for the force, and indeed a prescription using spheres, like that used by Chandrasekhar for gravity, is employed. We note that [25] focusses on the power-law tails associated with the singularity at zero separation of the force, which lead in this case (as can be inferred from the result summarized above) to the divergence of the first moment of the force PDF. One of us (AG) has given results previously [26] for the PDF for a generic power-law interaction in $d=1$ for $\gamma>-1$ in our notation above. The conditional force PDF is then derived for the case of an infinite "shuffled lattice" of particles, i.e., particles initially on an infinite lattice and then subjected to uncorrelated displacements of finite variance, and using again, as Chandrasekhar, a "spherical top-hat" prescription for the force summation (for $\gamma \leq 0$, when the pair force is not absolutely integrable). It is simple to show [18] that such a distribution has an SF with $n=2$ at small $k$, and thus the existence of the force PDF in these cases is again in line with the constraint (19) derived. Power-law tails are again observed in these cases, and their exponents related explicitly to the singularity in the assumed power-law force at zero separation.

The calculation of Chandrasekhar has been generalized in [27] to the case of particles on an infinite shuffled lattice. This leads again, in line with condition (19), to a well defined PDF, again with or without power-law tails according to whether the singularities in the pair force are included or not. Chavanis [31] considers, on the other hand, the generalization of Chandrasekhar calculation (for the PDF of gravitational forces in a Poisson distribution) to $d=2$ and $d=1$. The condition (19) for gravity $(\gamma=d-2)$ gives $n>-d+2$, which implies that the force PDF is not defined in the infinite system limit we have considered for $d \leq 2$, and indeed in [31] well defined PDFs are obtained in $d=2$ and $d=1$ by using a different limiting procedure involving in each case an appropriate rescaling of the coupling with $N$. The physical meaning of such a procedure is discussed in [22], which considers in detail the calculation of the force PDF for gravity in $d=1$ in a Poisson distribution (as in [31] ). An exact calculation of the force PDF of the screened gravitational force in the infinite system limit is given, which allows one to see in this case exactly how the general result given here is verified in this specific case: all moments of the PDF diverge simultaneously as the screening length is taken to infinity, giving a PDF which converges point-wise to zero. The force PDF for gravity in $d=1$ for a class of infinite particle distributions generated by perturbing a lattice has been derived recently by three of us in [32]. It is straightforward to show that one of the conditions imposed on the perturbations to obtain the PDF, that the variance of the perturbations be finite, corresponds in fact to the condition $n>1$ which coincides precisely with the more general condition (19) derived here. Unlike in the other specific cases just discussed, it turns out that in this case (gravity in $d=1$ ) it is in fact necessary to use the smooth prescription Eq. (17). As explained in detail in [32], the top-hat prescription does not give a well defined result in this case, because surface contributions to the force which do not decay with distance in this case are not regulated by it. We underline that the general result given in the present article are for this specific prescription Eq. (17). Further analysis would be required to derive the general conditions in which a top-hat prescription also gives the same (and well-defined) PDF.

Finally let us comment on why we anticipate the classification of pair interactions according to their "dynamical range", formalized here using the force $\mathrm{PDF}$, should be a useful and relevant one physically in the study of systems with long-range interactions. The reason is that this classification reflects, as we have explained, the relative importance of the mean field contribution to the force on a particle, due to the bulk, compared with that due to nearby particles. Now it is precisely the domination by the former which is understood to give the regime of collisionless dynamics which is expected to lead to the formation of QSS states, which are usually interpreted to be stationary states of the Vlasov equations describing such a regime of the dynamics (see e.g. [19]). In a recent article [30] by three of us, we have reported a numerical and analytical study which provides strong evidence for the following result, very much in line with this naive expectation: systems of particles interacting by attractive power law pair interactions like those considered here can always give rise to QSS; however when the pair force is dynamically short-range their existence requires the presence of a sufficiently large soft core, while in the dynamically long-range case QSS can occur independently of the core, whether hard or soft, provided it is sufficiently small. In other words only in the case of a pair force which is "dynamically long-range" can the occurrence of QSS be considered to be the result only of the long distance behavior of the interaction alone. This finding is very consistent with what could be anticipated from the preceding (naive) argument: the effect of a "soft core" is precisely to reduce the contribution to the force 
due to nearby particles, which would otherwise dominate over the mean field force in the case of a pair force which is absolutely integrable at large distances. Indeed the meaning of "sufficiently large" specified in [30] is that the size of the soft core must increase in an appropriate manner with the size of the system as the limit $N \rightarrow \infty$ is taken, while we have always implicitly assumed it to be fixed in units of the interparticle distance here. Further work on these issues will be reported elsewhere.

We thank M. Kiessling and T. Worrakitpoonpon for useful conversations, and P. Viot for useful comments on the manuscript.

\section{Appendix A: One and two point properties of uniform SPP}

In this appendix we give the general one and two-point statistical characterization of a SPP which is uniform on large scales.

The description of the correlation properties of a generic uniform SPP is given by the $n$-point correlation functions of the density field. For our considerations it will turn out to be sufficient to consider only the two-point properties, and more specifically it will be most convenient to characterize them in reciprocal space through the structure factor (SF) (or power spectrum). This is defined by

$$
S(\mathbf{k})=\lim _{V \rightarrow \infty} \frac{\left\langle|\tilde{\delta} n(\mathbf{k} ; V)|^{2}\right\rangle}{n_{0} V}
$$

where

$$
\tilde{\delta} n(\mathbf{k} ; V)=\int_{V} d^{d} x e^{-i \mathbf{k} \cdot \mathbf{x}}\left[n(\mathbf{x})-n_{0}\right] .
$$

With these normalisations the SF of an uncorrelated Poisson process is $S(\mathbf{k})=1$. For a statistically isotropic point process $S(\mathbf{k}) \equiv S(k)$, where $k=|\mathbf{k}|$. We recall here that $S(\mathbf{k})$ is the Fourier transform $(\mathrm{FT})$ of the connected two point density correlation function:

$$
S(\mathbf{k})=\int d^{d} x e^{-i \mathbf{k} \cdot \mathbf{x}} C(\mathbf{x})
$$

where

$$
C(\mathbf{x})=\frac{\left\langle n\left(\mathbf{x}_{0}+\mathbf{x}\right) n\left(\mathbf{x}_{0}\right)\right\rangle-n_{0}^{2}}{n_{0}}=\delta(\mathbf{x})+n_{0} h(\mathbf{x}) .
$$

In the last expression we have explicitly separated in the correlation function $C(\mathbf{x})$ the shot noise term $\delta(\mathbf{x})$, present in all SPP and due to the "granularity" of the particle distribution, from the "off-diagonal" term $n_{0} h(\mathbf{x})$ which gives the actual spatial correlations between different particles.

In the paper we study the convergence properties of forces at large distances and are thus mainly interested in the properties of the SF at small $k$. In this respect we will use the following limit on the SF which follows from the assumed uniformity of the SPP:

$$
\lim _{k \rightarrow 0} k^{d} S(\mathbf{k})=0,
$$

i.e, the $\mathrm{SF}$ is an integrable function of $\mathbf{k}$ at $k=0$. This constraint simply translates in reciprocal space the requirement from uniformity on the decay of relative fluctuations of the number of particles contained in a volume $V$ about the mean at large $V$ :

$$
\lim _{V \rightarrow \infty} \frac{\left\langle N(V)^{2}\right\rangle-\langle N(V)\rangle^{2}}{\langle N(V)\rangle^{2}}=0 .
$$

Given that $\langle N(V)\rangle \propto V$, the root mean square fluctuation of particle number $N$ in a volume $V$ must diverge slower than the volume $V$ itself in order that this condition be fulfilled. (This is equivalent to saying that $C(\mathbf{x})$ must vanish at large $x$ ).

We use likewise in the paper only one constraint on the large $k$ behavior of the SF, which is valid for any uniform SPP (see e.g. [18]) and coincides with the shot noise term in the correlation function $C(\mathbf{x})$ :

$$
\lim _{k \rightarrow \infty} S(\mathbf{k})=1
$$

\section{Appendix B: Small k behavior of $\tilde{\mathbf{f}}(\mathbf{k})$}

We are interested in the small $k$ behavior of the Fourier transform $\tilde{\mathbf{f}}(\mathbf{k})$ of the pair force in $d$ dimensions in the case where the pair force $\mathbf{f}(\mathbf{x})=\hat{\mathbf{x}} f(x)$, where $\hat{\mathbf{x}}=\frac{\mathbf{x}}{|\mathbf{x}|}$, is non-integrable but converges to zero at $x \rightarrow \infty$, i.e., $f(r) \sim x^{-(\gamma+1)}$ at large $x$ with $-1<\gamma \leq d-1$.

We first show that for a function $\mathbf{f}(\mathbf{x})=\hat{\mathbf{x}} f(x)$, its Fourier transform, $\tilde{\mathbf{f}}(\mathbf{k})=\mathrm{FT}[\mathbf{f}(\mathbf{x})](\mathbf{k})$, can be written $\tilde{\mathbf{f}}(\mathbf{k})=\hat{\mathbf{k}} \psi(k)$ where $\psi(k)$ is a function depending only on the modulus of $\mathbf{k}$ and $\hat{\mathbf{k}}=\frac{\mathbf{k}}{|\mathbf{k}|}$. In order to obtain this result, we start by writing

$$
\tilde{\mathbf{f}}(\mathbf{k})=\int d^{d} x \mathbf{f}(\mathbf{x}) e^{-i \mathbf{k} \cdot \mathbf{x}}=\int d^{d} x \hat{\mathbf{x}} f(x) e^{-i \mathbf{k} \mathbf{x}},
$$

where this integral is defined in the sense of functions or distributions according to the integrability of $f(x)$.

In the following we denote by $\left(\hat{\mathbf{e}}_{1}, \hat{\mathbf{e}}_{\mathbf{2}}, \ldots, \hat{\mathbf{e}}_{\mathbf{n}}\right)$ the cartesian vector basis in $d$-dimension and we define $\left(r, \theta_{1}, \theta_{2}, \ldots, \theta_{d-1}\right)$ the hyper-spherical coordinates of $\mathbf{x}$. Considering $\mathbf{k}=k \hat{\mathbf{e}}_{\mathbf{1}}$ and denoting for simplicity $\theta=\theta_{1}$, we can write

$$
\tilde{\mathbf{f}}(\mathbf{k})=\int d^{d} x \hat{\mathbf{x}} f(x) e^{-i k x \cos \theta},
$$

where

$$
d^{d} x=\left(\prod_{j=0}^{d-1} \sin ^{j}\left(\theta_{d-j}\right) d \theta_{d-j}\right) x^{d-1} d x
$$


Projecting $\tilde{\mathbf{f}}(\mathbf{k})$ on the cartesian basis, it is easy to see that the only non-vanishing term is $\hat{\mathbf{e}}_{\mathbf{1}} \tilde{\mathbf{f}} \tilde{\mathbf{f}}(\mathbf{k})$ which gives

$$
\begin{aligned}
& \hat{e}_{1} \cdot \tilde{f}(\mathbf{k})=C_{\theta_{i \neq 1}} \int_{0}^{\infty} d x x^{d-1} \\
& \times \int_{0}^{\pi} d \theta \sin ^{n-2}(\theta) \cos \theta f(x) e^{-i k x \cos \theta},
\end{aligned}
$$

where $C_{\theta_{i \neq 1}}$ is a constant term coming from the integration over all the hyper-spherical coordinates $\theta_{i}$ with $i \neq 1$. We thus can write $\tilde{\mathbf{f}}(\mathbf{k})=\hat{\mathbf{k}} \psi(k)$ where $\psi(k)$ is a function depending only on the modulus of $\mathbf{k}$.

We now focus our attention on the small $k$ behavior of the term

$$
\int_{0}^{\infty} d x x^{d-1} f(r) e^{-i k x \cos \theta}
$$

where the function $f(x)$ is non-integrable but converges to zero at $x \rightarrow \infty$, i.e., $f(x) \sim x^{-(\gamma+1)}$ at large $x$ with $-1<\gamma \leq d-1$, and thus can be written $f(x)=$ $x^{-(\gamma+1)}+h(x)$ with $h(x)$ a smooth function, integrable at $x=0$ and such that $x^{\gamma+1} h(x) \rightarrow 0$ for $x \rightarrow \infty$.

Defining explicitly eq. (B1) in the sense of distributions, the small $k$ behavior is determined by this leading divergence at $x \rightarrow \infty$,

$$
\lim _{\mu \rightarrow 0} \int_{0}^{\infty} d x x^{d-1} \frac{e^{-\mu x}}{x^{\gamma+1}} e^{-i k x \cos \theta}
$$

where the parameter $\mu>0$. We define $\alpha=d-\gamma-2$ which satisfies $-1 \leq \alpha<d-1$ and rewrite eq. (B2)

$$
\lim _{\mu \rightarrow 0} \int_{0}^{\infty} d x x^{\alpha} e^{-(i k \cos \theta+\mu) x}
$$

This can be easily calculated with Laplace's transform and gives

$$
\int_{0}^{\infty} d x x^{\alpha} e^{-(i k \cos \theta+\mu) x}=\frac{\Gamma(\alpha+1)}{(\mu+i k \cos \theta)^{\alpha+1}} .
$$

We can conclude that

$$
\begin{aligned}
& \lim _{\mu \rightarrow 0} \int_{0}^{\infty} d x x^{d-1} \frac{e^{-\mu x}}{x^{\gamma+1}} e^{-i k x \cos \theta} \\
& =i^{-(\alpha+1)} \cos ^{-(\alpha+1)}(\theta) \Gamma(\alpha+1) k^{-(\alpha+1)} \sim k^{\gamma-d+1} .
\end{aligned}
$$

[1] D. Ruelle, Statistical Mechanics: Rigorous results (W. A. Benjamin, 1983).

[2] T. Dauxois, S. Ruffo, E. Arimondo, and M. Wilkens, Dynamics and Thermodynamics of Systems with Long Range Interactions (Springer, Berlin, 2002).

[3] A. Campa, A. Giansanti, G. Morigi, and F. Sylos Labini, Dynamics and Thermodynamics of Systems with Long Range Interactions: Theory and experiments (AIP Conference Proceedings, 2008).

[4] P. H. Chavanis, Int. Jour. Mod. Phys. B 20, 3113 (2006).

[5] W. Thirring, Quantum Mathematical Physics (Springer, 2002).

[6] K. R. Yawn and B. N. Miller, Phys. Rev. E 68, 056120 (2003).

[7] Y. Y. Yamaguchi, J. Barré, F. Bouchet, T. Dauxois, and S. Ruffo, Physica A 337, 36 (2004), cond-mat/0312480.

[8] A. Antoniazzi, F. Califano, D. Fanelli, and S. Ruffo, Phys. Rev. Lett. 98, 150602 (2007).

[9] A. Antoniazzi, D. Fanelli, S. Ruffo, and Y. Y. Yamaguchi, Phys. Rev. Lett. 99, 040601 (2007).

[10] Y. Y. Yamaguchi, Phys. Rev. E 78, 041114 (2008).

[11] J. Binney and S. Tremaine, Galactic Dynamics (Princeton University Press, 1994).

[12] A. Campa, A. Giansanti, and G. Morelli, Phys. Rev. E76, 041117 (2007), arXiv:0706.3664.

[13] F. Baldovin, P. Chavanis, and E. Orlandini, Phys. Rev. E79, 011102 (2009), cond-mat/0501583.

[14] P. Chavanis, Journal of Statistical Mechanics: Theory and Experiment 5, 19 (2010), 1002.3268.

[15] T. N. Teles, Y. Levin, R. Pakter, and F. B. Rizzato, J. Stat. Mech. 5, 7 (2010), 1004.0247.

[16] S. Gupta and D. Mukamel, Phys. Rev. Lett. 105, 040602 (2010), arXiv:1006.0233.
[17] S. Chandrasekhar, Rev. Mod. Phys. 15, 1 (1943).

[18] A. Gabrielli, F. Sylos Labini, M. Joyce, and L. Pietronero, Statistical Physics for Cosmic Structures (Springer, 2004).

[19] R. Balescu, Equilibrium and nonequilibrium statistical mechanics (New York: Wiley, 1975, 1975).

[20] O.E. Lanford III, Commun. math. Phys. 9, 176 (1968).

[21] O.E. Lanford III, Commun. math. Phys. 11, 257 (1969).

[22] A. Gabrielli and M. Joyce, Phys. Rev. E 81, 021102 (2010), 0909.5034.

[23] M. K.-H. Kiessling, Adv. Appl. Math. 31, 132 (2003), astro-ph/9910247.

[24] J. H. Jeans, Phil. Trans. Roy. Soc. 199, 1 (1902).

[25] J. H. Wesenberg and K. Mølmer, Phys. Rev. Lett. 93, 143903 (2004), arXiv:quant-ph/0406178.

[26] A. Gabrielli, Phys. Rev. E. 72, 066113 (2005), arXiv:cond-mat/0506365.

[27] A. Gabrielli, T. Baertschiger, M. Joyce, B. Marcos, and F. S. Labini, Phys. Rev. E 74, 021110 (2006), arXiv:condmat/0603124.

[28] A. Kolmogorov and S. Fomin, Elements of the Theory of Functions and Functional Analysis (Dover Publications, New York, 1999).

[29] P. J. E. Peebles, The Large-Scale Structure of the Universe (Princeton University Press, 1980).

[30] A. Gabrielli, M. Joyce, and B. Marcos (2010), arXiv: 1004.5119

[31] P. H. Chavanis, European Physical Journal B 70, 413 (2009), 0810.1211.

[32] A. Gabrielli, M. Joyce, and F. Sicard, Phys. Rev. E80, 041108 (2009). 